\title{
High-reflectivity high-resolution X-ray crystal optics with diamonds
}

\author{
Yuri V. Shvyd'ko ${ }^{1 \star}$, Stanislav Stoupin ${ }^{1}$, Alessandro Cunsolo ${ }^{1,2}$, Ayman H. Said' and Xianrong Huang ${ }^{2}$
}

Owing to the depth to which hard X-rays penetrate into most materials, it is commonly accepted that the only way to realize hard-X-ray mirrors with near $100 \%$ reflectance is under conditions of total external reflection at grazing incidence to a surface. At angles away from grazing incidence, substantial reflectance of hard $X$-rays occurs only as a result of constructive interference of the waves scattered from periodically ordered atomic planes in crystals (Bragg diffraction). Theory predicts that even at normal incidence the reflection of $X$-rays from diamond under the Bragg condition should approach $100 \%$-substantially higher than from any other crystal. Here we demonstrate that commercially produced synthetic diamond crystals do indeed show an unprecedented reflecting power at normal incidence and millielectronvolt-narrow reflection bandwidths for hard X-rays. Bragg diffraction measurements of reflectivity and the energy bandwidth show remarkable agreement with theory. Such properties are valuable to the development of hard-X-ray optics, and could greatly assist the realization of fully coherent $\mathrm{X}$-ray sources, such as $\mathrm{X}$-ray free-electron laser oscillators ${ }^{1-3}$.

Diamond is a material with superlative physical qualities: high mechanical hardness, high thermal conductivity, high dispersion index, high radiation hardness, high hole and electron mobilities, low thermal expansion and chemically inert ${ }^{4}$. Technological applications of diamond crystals are increasing not only in the traditional fields of cutting, grinding and polishing tools, but also in high-tech applications, such as diamond-based electronic devices, wide-bandgap radiation detectors, ultraviolet-emitting diodes, biochemical sensors, high-pressure cells and thermal sinks, to name only a few. Very recently, diamond crystals have been identified as indispensable for the realization of X-ray free-electron laser oscillators (XFELOs), next-generation hard-X-ray sources of the highest average and peak brightness and extremely narrow bandwidth $^{1,2}$. The special role of diamonds in the feasibility of the XFELOs is due to their outstanding reflectivity for hard X-rays in Bragg diffraction, thus far only predicted in theory (Fig. 1a).

The high reflectivity of crystals in Bragg diffraction is intimately connected with the perfect crystal structure. Progress in fabrication, characterization and X-ray optics applications of synthetic diamonds was substantial in the past decade $e^{5-13}$. Still the diamond crystals available commercially as a rule suffer from defects: dislocations, stacking faults, inclusions, impurities and so on. Synthetic high-purity (type IIa, low nitrogen content) crystals grown with a high-pressure, high-temperature technique are generally considered to have the highest crystal quality and the lowest density of defects among commercially available diamonds ${ }^{10,13}$. $\mathrm{X}$-ray topography studies have demonstrated crystals with relatively large $\simeq 4 \times 4 \mathrm{~mm}^{2}$ defect-free areas ${ }^{13}$. However, critical outstanding questions remain open. Can the remarkably high reflectivity of a

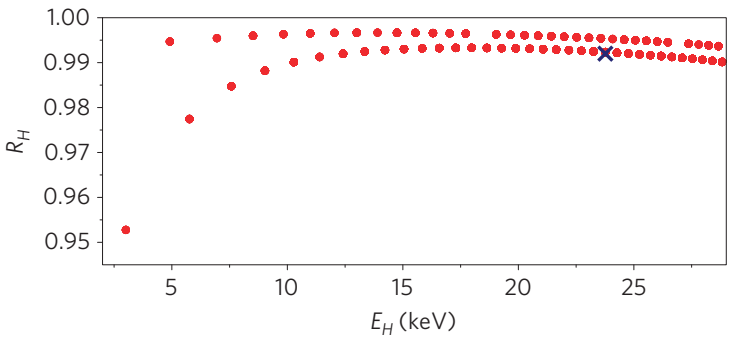

b
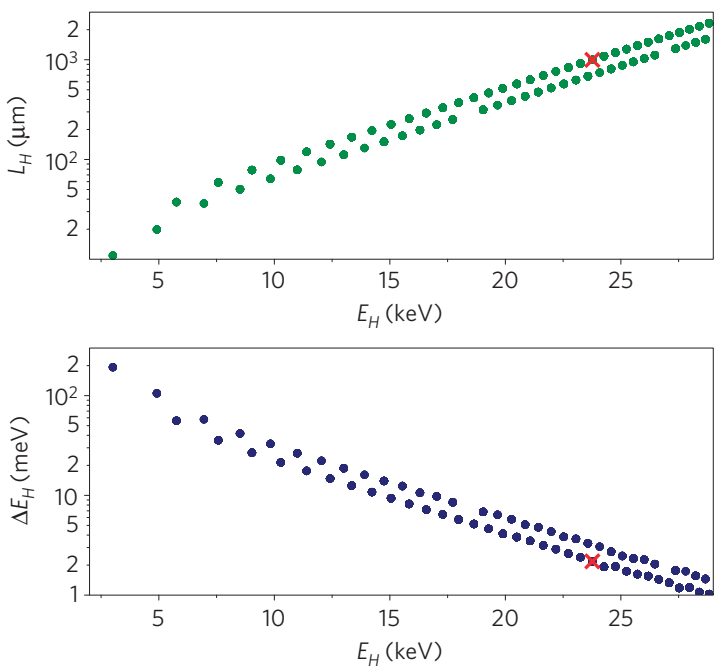

Figure 1 | Calculated peak reflectivity, interaction length and reflection energy bandwidth for $\mathrm{X}$-rays in Bragg backscattering from diamond for all allowed reflections with Bragg energies $E_{\boldsymbol{H}}$ up to $\mathbf{3 0} \mathbf{k e V}$. a, Peak reflectivity $R_{H} . \mathbf{b}$, Crystal thickness $L_{H}$ required to attain the peak reflectivity $R_{H}$. c, Energy width $\Delta E_{H}$. The upper set of points in $\mathbf{a}$ and $\mathbf{c}$ and the lower set in $\mathbf{b}$ represent the even Bragg reflections in diamond. The complementary set of points represents the odd reflections. The crosses indicate the theoretical results for the (995) Bragg reflection used in the experiment. Calculations are carried out using the dynamical theory of $\mathrm{X}$-ray diffraction in thick crystals, as described, for example, in ref. 14.

diamond crystals that is predicted in theory be achieved in practice? Is the quality of the diamond crystals available at present sufficiently high for practical use as high-reflectivity X-ray mirrors for XFELOs and other highly advanced technology applications relying on high crystal perfection?

Here, we show that commercially available synthetic diamond crystals, of type IIa, have a high degree of perfection with $\approx 10^{7}$ perfectly arranged, strictly periodic crystallographic planes of atoms. Almost theoretical values were measured for the spectral

${ }^{1}$ Advanced Photon Source, Argonne National Laboratory, Argonne, Illinois 60439, USA, ${ }^{2}$ NSLS-II, Brookhaven National Laboratory, Upton, New York 11973 , USA. *e-mail: shvydko@aps.anl.gov. 

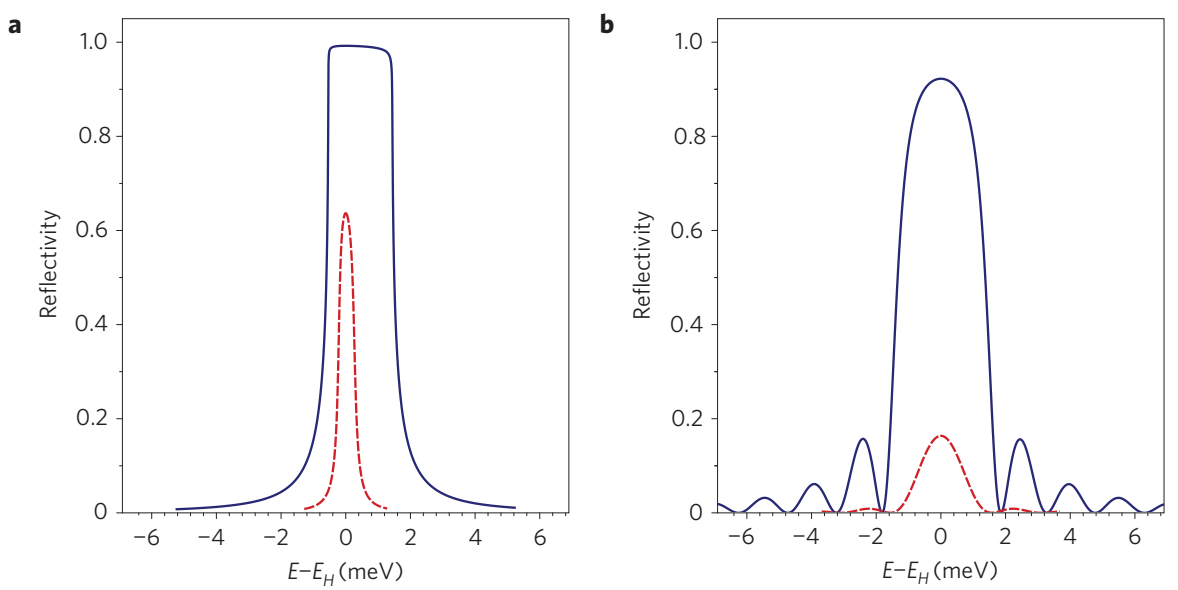

Figure 2 | Reflectivity of X-rays in backscattering as a function of photon energy, according to dynamical theory ${ }^{\mathbf{1 4}, \mathbf{1 9}}$ calculations. Solid lines: the (995) Bragg reflection in diamond, $E_{H}=23.77 \mathrm{keV}$. Dashed lines: the (19 75) Bragg reflection in Si, with a comparable Bragg energy $E_{H}=23.80 \mathrm{keV}$. a, Thick crystals $d \gg L_{H}$. b. Crystal thickness $d=0.4 \mathrm{~mm}$, as in the experiment.

width $\Delta E=2.9 \mathrm{meV}$, and for the reflectivity $R=89 \%$, in Bragg diffraction at normal incidence to the reflecting atomic planes of hard X-rays with photon energy $E=23.7 \mathrm{keV}$. The reflectivity is several times higher than the reflectivity of Si crystals under similar conditions. These findings open up vistas for entirely new highly advanced technology applications of diamonds and new opportunities in X-ray optics, in particular.

Total (100\%) reflection is achieved in Bragg diffraction of X-rays from crystals provided, first, there are no losses in the crystal owing to, for example, photoabsorption, and, second, the crystal is sufficiently thick. Bragg diffraction is a coherent scattering process from periodically arranged atomic planes. The extinction length $L_{H}^{\text {ext }}$ measures the interaction depth of X-rays with the crystal lattice in Bragg diffraction. It is an invariant value for each Bragg reflection. Typically a crystal thickness of $\simeq 10 L^{\text {ext }}$ is required to achieve total reflectivity ${ }^{14}$. Figure $1 \mathrm{~b}$ shows the dynamical theory calculations for $L_{H}=10 L_{H}^{\text {ext }}$ for all allowed Bragg reflections in diamond, defined by the diffraction vector $\mathbf{H} \quad\left(H=2 \pi / d_{H}, d_{H}\right.$ is the interplanar distance). For a larger $H$, that is, for a larger Bragg energy $\left(E_{H}=h c / 2 d_{H}\right.$ - photon energy at backscattering, with $h$ as the Planck constant and $c$ as the speed of light in vacuum), thicker crystals are required to achieve the maximum reflectivity (Fig. 1b).

The thicker the crystal, the smaller the energy width $\Delta E$ of the Bragg reflection (Fig. 1c). The relative energy width $\Delta E / E$ of the Bragg reflection is

$$
\frac{\Delta E}{E} \simeq \frac{1}{N_{d}}
$$

where $N_{d}=d / d_{H}$ is the number of atomic planes through crystal thickness $d$ (ref. 14). Thus, $E / \Delta E$ measures the quantity of the perfectly arranged atomic planes in the crystal contributing to Bragg diffraction; $\Delta E / E$ is a fingerprint of the crystal perfection.

In reality, photoabsorption always exists, and $100 \%$ reflectivity is never achievable. However, the peak reflectivity could be close to $100 \%$ provided the photoabsorption length $L^{\mathrm{ph}}$ in the crystal is much larger than the extinction length: $L^{\mathrm{ph}} \gg L_{H}^{\text {ext }}$. This is fulfilled for crystals with a high Debye temperature and composed of low- $Z$ atoms. The high Debye temperature ensures rigidity of the crystal lattice, and thus the smaller number of atomic planes required for maximum reflectivity. The low $Z$ ensures larger photoabsorption lengths. Diamond, $\mathrm{Be}, \mathrm{BeO}, \mathrm{Al}_{2} \mathrm{O}_{3}, \mathrm{SiC}$ and so on are among attractive crystals. However, diamond, owing to the superlative qualities mentioned above, is most favourable. Figure 1a shows the peak reflectivity values in diamond in Bragg backscattering.
More than $99 \%$ reflectivity is predicted by the dynamical theory calculations for Bragg reflections with $E_{H}>10 \mathrm{keV}$. Debye-Waller factors are calculated using a Debye temperature of 2,200 K. The results shown in Fig. 1 were calculated for backscattering, which is a representative case, with the smallest reflectivity for the given Bragg reflection.

To probe larger crystal depths, reflections with large Bragg energies $E_{H}$ have to be used. In the present studies, the (995) Bragg reflection is used with $E_{H}=23.765 \mathrm{keV}$. To achieve the theoretical reflectivity of $R_{H}=0.99$, a crystal with $d \geq L_{H} \simeq 1 \mathrm{~mm}$ thickness is required. Such a crystal should show a Bragg reflection spectral width of $\Delta E_{H}=2.3 \mathrm{meV}$ (Fig. 2a). The dashed line in Fig. 2a shows for comparison the reflectivity from $\mathrm{Si}$, the (1975) Bragg reflection, for photons with a similar energy. The Debye temperature of Si $(\simeq 540 \mathrm{~K})$, which is smaller than in diamond, reflects a less rigid atomic lattice, requiring more atomic planes (thicker crystals: $d>L_{H} \simeq 3 \mathrm{~mm}$ ) to attain maximum reflectivity. In silicon, $L_{H}$ becomes comparable to the photoabsorption length $L^{\mathrm{ph}}$ and results in a significantly lower reflectivity, as compared with that of diamond.

A typical size of crystals used in these studies is $\simeq 8 \times 4 \mathrm{~mm}^{2}$ with a thickness $d \simeq 0.4 \mathrm{~mm}$. The latter is, however, smaller than $L_{H}$ of the (995) Bragg reflection. For diamonds with $d \simeq 0.4 \mathrm{~mm}$, theory predicts a larger value for the spectral width $\Delta E_{H}(d)=2.8 \mathrm{meV}$, and a smaller although still very high value for peak reflectivity $R_{H}(d)=0.92$ (Fig. 2b). The dashed line in Fig. 2b shows a much lower reflectivity of Si of the same thickness for comparison.

A white-beam X-ray topogram of a selected diamond is shown in Fig. 3a. The image is produced by the (1)13) Bragg reflection and indicates that the crystal is of relatively high quality in most of the central and upper regions. The main defects in these regions include a few dislocations (fine black lines), a relatively large stacking fault (the black ribbon) and some small stacking faults close to the dislocations. In the central part, there are a few millimetre-squared areas free of defects. In the lower part of the crystal, the crystalline quality is worse with stacking faults and dislocations mixed together.

In the next step, the Bragg reflectivity of the selected crystal was measured with highly monochromatic X-rays as a function of photon energy in nearly exact backscattering geometry. Figure 4 shows the experimental set-up. X-ray photons with energies close to the Bragg energy $E_{H}=23.765 \mathrm{keV}$ of $\mathrm{C}(995)$ are monochromatized to a bandwidth $\Delta E_{\mathrm{x}} \simeq 1 \mathrm{meV}$, which is smaller than the expected energy width $\Delta E_{H}(d)=2.8 \mathrm{meV}$ of the $\mathrm{C}(995)$ Bragg reflection.

Reflectivity curves as a function of the X-ray photon energy are measured with the X-ray beam illuminating different parts of 


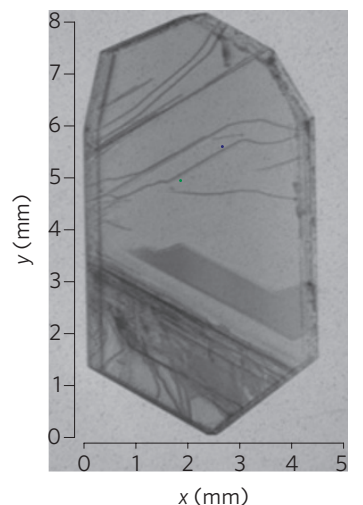

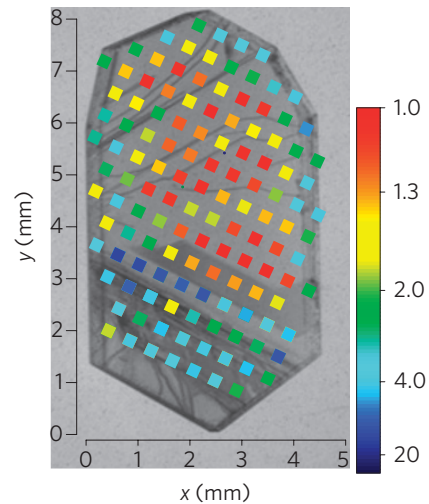

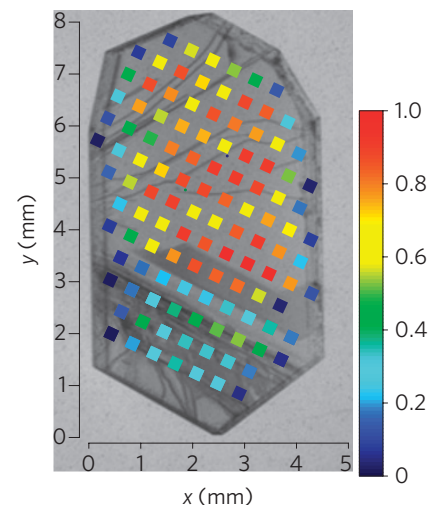

Figure 3 | X-ray images of the sample diamond crystal. a, White-beam X-ray topogram of the diamond crystal plate in transmission. $\mathbf{b}, \mathbf{c}$, Colour maps for the relative spectral width, $\Delta E / \Delta E_{\min }(\mathbf{b})$, and the relative peak reflectivity for the (995) Bragg reflection, $R / R_{\max }$ (c), at different points on the diamond crystal, measured with a $23.765 \mathrm{keV} \mathrm{X-ray} \mathrm{photon} \mathrm{beam} \mathrm{of} 0.7 \times 0.7 \mathrm{~mm}^{2}$ cross-section. The beam footprint is larger than the pixels on the colour maps, and has been reduced in size to allow for direct comparison with the underlying white-beam topogram.

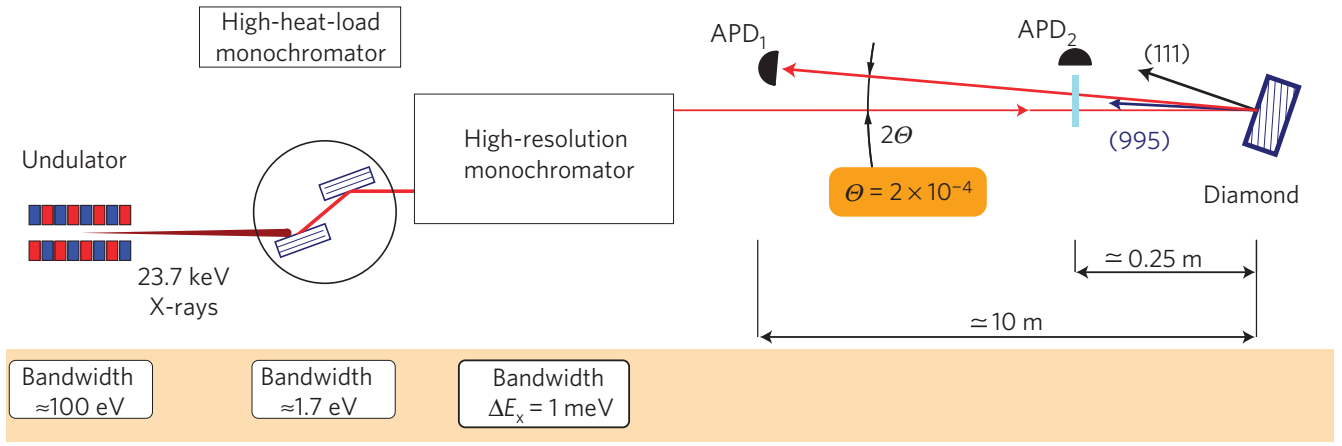

Figure 4 | Experimental set-up. (See the Methods section for details.) The diamond reflectivity measurements were carried out with $E=23.7 \mathrm{keV} X$-rays. An extremely highly monochromatic $X$-ray beam was used with a bandwidth of $\Delta E_{\mathrm{x}} \simeq 1 \mathrm{meV}$, smaller than the energy width of the studied $\mathrm{C}(995)$ reflection. The monochromatization is achieved by successive applications of two $\mathrm{X}$-ray monochromators: the high-heat-load diamond monochromator and a Si HRM. The highly monochromatic beam impinges on the diamond crystal, placed at a distance $\simeq 10 \mathrm{~m}$ downstream from the HRM. The (995) planes in the diamond crystal are oriented with a very small angular offset $\Theta \simeq 4 \times 10^{-4}$ rad from normal incidence. X-rays are reflected with a scattering angle deviating from $180^{\circ}$ by $2 \Theta$ and detected by an avalanche photodiode APD . The small deviation $\Theta$ from normal incidence ensures a negligible contribution of the angular spread in the incident beam on the reflectivity measurements. The $\mathrm{APD}_{2}$ detector is used to measure the absolute reflectivity.

the crystal. An avalanche photodiode $\mathrm{APD}_{1}$ is used as the X-ray detector. The colour maps of the relative energy widths and relative peak reflectivities are shown in Fig. $3 \mathrm{~b}$ and $\mathrm{c}$, respectively. The results reveal an inhomogeneous quality of the crystal. However, in agreement with the topography studies, the regions with a small defect density feature a narrow energy width and high reflectivity (red pixels), that is, high perfection.

The filled circles in Fig. 5a show an experimental energy dependence of the Bragg reflectivity, representative among those with the narrowest energy width. The dependence is plotted on a logarithmic scale. Figure $5 \mathrm{a}$, inset, shows the same dependencies on a linear scale. The energy width is $\Delta E=2.9 \mathrm{meV}$, which matches perfectly the expected $\Delta E_{H}(d)=2.8 \mathrm{meV}$, if the photon energy spread $\Delta E_{\mathrm{x}}=1 \mathrm{meV}$ is also taken into account. The oscillations on the wings in Fig. $5 \mathrm{a}$ are due to the interference of the waves reflected from the front and the rear crystal surfaces. This interference is another fingerprint of high crystal quality. The period is $E_{d}=h c / 2 d$ (ref. 14). This simple relation allows one to directly ascertain the crystal thickness to be $d=415(5) \mu \mathrm{m}$. The dotted line in Fig. 5a shows the results of the calculations of the reflectivity for a $d=415(5)-\mu$ m-thick crystal, for perfectly monochromatic X-rays. The solid line represents similar calculations, assuming a $1 \mathrm{meV}$ broad Gaussian distribution of X-ray photon energies, as in the experiment. The experimental curve in Fig. 5a is scaled to best fit the tails of the theoretical curve. The scaling factor is the only free parameter. Comparison shows a remarkable agreement between the measured and calculated dependencies, in the shape and the energy width. The only difference is that the peak value $\simeq 0.87(1)$ of the scaled experimental curve is slightly smaller than the peak reflectivity of $R_{H}(d)=0.91$, expected in theory.

Direct measurements of the absolute reflectivity are carried out using detector $\mathrm{APD}_{2}$. It counts the photons scattered from a doublelayered $2 \times 20 \mu \mathrm{m}$ low-absorbing $(\simeq 0.2 \%)$ Scotch Magic Tape, installed in the incident and reflected beams $0.25 \mathrm{~m}$ upstream from the diamond crystal (see Fig. 4). A value proportional to the net intensity $I(E)=I_{0}+I_{R}(E)$ of X-ray photons incident on the crystal and reflected from the crystal is measured. The absolute crystal reflectivity is then determined as $R(E)=\left[I(E)-I_{0}\right] /\left[I_{0}(1-A)\right]$, assuming that $I_{0}=I(E)$ when the photon energy is detuned from the reflection peak: $E \simeq E_{H} \pm 20 \mathrm{meV}$. Taking into account absorption of photons propagating $0.5 \mathrm{~m}$ in air from the tape to the crystal and back, and in the tape, $A=0.029$. The filled circles in Fig. $5 \mathrm{~b}$ show the results of the measurement. The peak reflectivity is determined as $R=0.89 \pm 0.015$, which is very close to the expected $R_{H}(d)=0.91$ and is in agreement with the indirect measurements, shown in Fig. 5 a.

The high X-ray reflectivity of diamond combined with its stability under high-heat-load conditions, and the recently demonstrated 

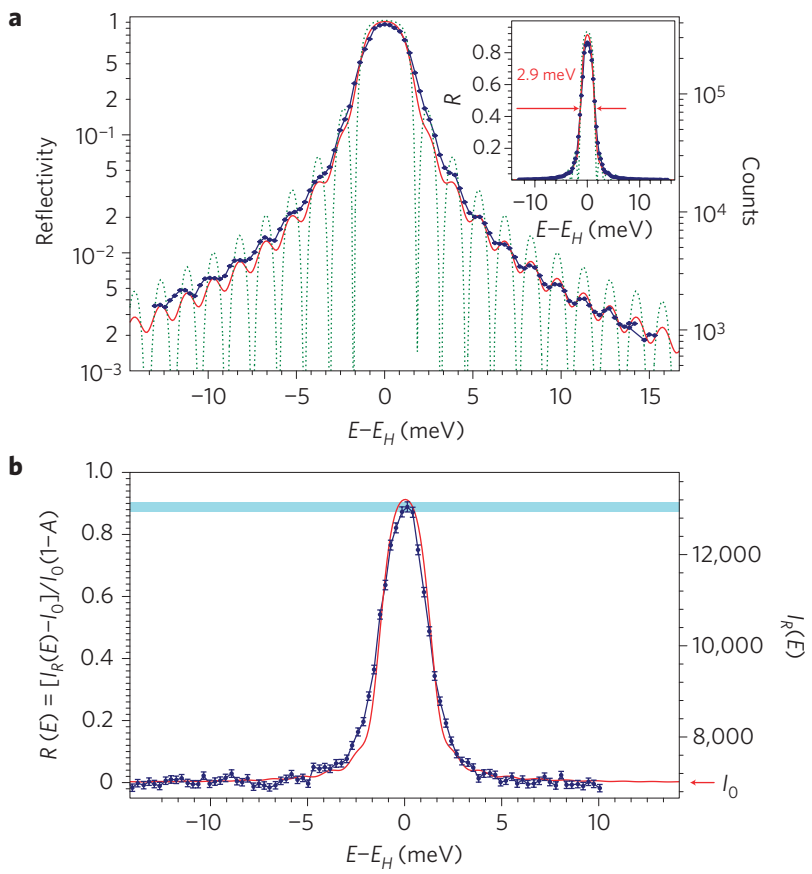

Figure 5 | Reflectivity of X-rays as a function of photon energy $E$ in Bragg backscattering from the (995) atomic planes in diamond, $\boldsymbol{E}_{\boldsymbol{H}}=\mathbf{2 3 . 7 6 5 ~ k e V . ~ a , ~ F i l l e d ~ c i r c l e s : ~ e x p e r i m e n t a l ~ d a t a . ~ D o t t e d ~ l i n e : ~}$ dynamical theory calculations for a $415-\mu \mathrm{m}$-thick crystal and perfectly monochromatic incident $X$-rays. Solid line: calculations for the $X$-rays with a $1 \mathrm{meV}$ bandwidth, as in the experiment. Inset: The same dependencies shown on a linear scale. $\mathbf{b}$, Results of the absolute reflectivity measurements.

low thermal expansion of less than $10^{-8} \mathrm{~K}^{-1}$ at temperatures $\lesssim 100 \mathrm{~K}$ (ref. 15) makes diamond potentially valuable in the development of high-efficiency, high-resolution X-ray optics for use in next-generation fully coherent X-ray sources, such as XFELOs (refs 1, 2) and seeded X-ray free-electron lasers ${ }^{16}$. Moreover, high-reflectance normal incidence diamond X-ray mirrors could enable the realization of high-finesse X-ray Fabry-Pérot interferometers ${ }^{14,17}$ - devices that will be useful as interference spectral filters (monochromators and analysers) for inelastic Xray scattering spectroscopy with an unprecedented narrow bandwidth $\Delta E \lesssim 100 \mu \mathrm{eV}\left(\Delta E / E \lesssim 10^{-8}\right)$ and large angular acceptance $2 \sqrt{\Delta E / E} \simeq 200 \mu \mathrm{rad}$.

\section{Methods}

The diamond reflectivity measurements were carried out with $E=23.765 \mathrm{keV}$ $\mathrm{X}$-rays at undulator beamline IXS/XOR 30-ID of the Advanced Photon Source. An extremely highly monochromatic X-ray beam was used with a bandwidth of $\Delta E_{\mathrm{x}} \simeq 1 \mathrm{meV}$, which is smaller than the energy width of the studied $\mathrm{C}(995)$ reflection, $\Delta E_{H}(d)=2.8 \mathrm{meV}$. The monochromatization is achieved by successive applications of two X-ray monochromators. First, the high-heat-load diamond monochromator selects photons with a $\simeq 1.7 \mathrm{eV}$ bandwidth. In the second step, a Si high-resolution monochromator ${ }^{18}$ (HRM) selects photons to a desired bandwidth of $\Delta E_{\mathrm{x}} \simeq 1 \mathrm{meV}$. The highly monochromatic beam $\Delta E_{\mathrm{x}} / E \simeq 4 \times 10^{-8}$ with an angular divergence of about $10 \mu \mathrm{rad}(\mathrm{V}) \times 20 \mu \mathrm{rad}(\mathrm{H})$, and a cross-section of $0.7 \times 0.7 \mathrm{~mm}^{2}$ impinges on the diamond crystal, placed at a distance $\simeq 10 \mathrm{~m}$ downstream from the HRM. The crystal temperature is $T=300 \mathrm{~K}$. The (995) planes in the diamond crystal are oriented with a very small angular offset $\Theta \simeq 4 \times 10^{-4}$ rad from normal incidence. X-rays are reflected with a scattering angle deviating from $180^{\circ}$ by $2 \Theta$ and detected by an avalanche photodiode $\mathrm{APD}_{1}$. The small deviation $\Theta$ from normal incidence ensures a negligible contribution of the angular spread in the incident beam on the reflectivity measurements.

Synchrotron white-beam topography was applied to select the crystal with the lowest density of defects, among the available samples. Imaging of the crystals was carried out at beamline X19C of the National Synchrotron Light Source, in transmission geometry.
A large interaction length of hard X-rays with crystals, typically more than $10 \mu \mathrm{m}$, see Fig. 1b, requires high perfection in the crystal volume rather than of the crystal surface. Diamond crystal plates with (111) orientation, of type IIa, are used in the studies, grown with a high-pressure, high-temperature technique by Sumitomo Electric Industries. Type IIa material has a low density of defects and less than $1 \mathrm{ppm}$ concentration of impurities. Such an amount of impurities most probably has a negligible influence on the measured properties, well below the detection limit of the extremely sensitive method used.

Received 8 September 2009; accepted 7 December 2009; published online 17 January 2010

\section{References}

1. Kim, K.-J., Shvyd'ko, Y. \& Reiche, S. An X-ray free-electron laser oscillator with an energy recovery linac. Phys. Rev. Lett. 100, 244802 (2008).

2. Kim, K.-J. \& Shvyd'ko, Y. Tunable optical cavity for an X-ray free-electron-laser oscillator. Phys. Rev. Phys. Spec. Top Accel. Beams 12, 030703 (2009).

3. Colella, R. \& Luccio, A. Proposal for a free electron laser in the X-ray region. Opt. Commun. 50, 41-44 (1984).

4. Prelas, M. A., Popovici, G. \& Bigelow, L. K. (eds) in Handbook of Industrial Diamonds and Diamond Films (Marcel Dekker, 1998).

5. Pal'yanov, Y., Malinovsky, Y., Borzdov, Y. M. \& Khokryakov, A. F. Use of the 'split sphere' apparatus for growing large diamond crystals without the use of a hydraulic press. Dokl. Akad. Nauk SSSR 315, 233-237 (1990).

6. Berman, L. E. et al. Diamond crystal X-ray optics for high-power-density synchrotron radiation beams. Nucl. Instrum. Methods Phys. Res. A 329, 555-563 (1993).

7. Sumiya, H. \& Satoh, S. High-pressure synthesis of high-purity diamond crystal. Diamond Relat. Mater. 5, 1359-1365 (1996).

8. Fernandez, P. et al. Test of a high-heat-load double-crystal diamond monochromator at the Advanced Photon Source. Nucl. Instrum. Methods Phys. Res. A 400, 476-483 (1997).

9. Sellschop, J. P. F. et al. Synchrotron X-ray applications of synthetic diamonds. New Diamond Frontier Carbon Technol. 10, 253-258 (2000).

10. Sumiya, H., Toda, N. \& Satoh, S. High-quality large diamond crystals. New Diamond Frontier Carbon Technol. 10, 233-251 (2000).

11. Zhong, Y. et al. Rocking curve FWHM maps of a chemically etched (001) oriented HPHT type Ib diamond crystal plate. J. Phys. D 40, 5301-5305 (2007).

12. Yabashi, M. et al. Diamond double-crystal monochromator for SPring-8 undulator beamlines. AIP Conf. Proc. 879, 922-925 (2007).

13. Burns, R. C. et al. HPHT growth and X-ray characterization of high-quality type IIa diamond. J. Phys. Condens. Matter 21, 364224 (2009).

14. Shvyd'ko, Y. X-ray Optics—High-Energy-Resolution Applications Vol. 98 (Optical Sciences, Springer, 2004).

15. Stoupin, S. \& Shvyd'ko, Y. V. Thermal expansion of diamond at low temperatures. Phys. Rev. Lett. (2009, in the press).

16. Saldin, E. L., Schneidmiller, E. A., Shvyd'ko, Y. V. \& Yurkov, M. V. X-ray FEL with a meV bandwidth. Nucl. Instrum. Methods Phys. Res. A 475, 375-362 (2001).

17. Shyyd'ko, Y. V. X-ray Resonators and Other Applications of Bragg Backscattering (Habilitationsschrift, DESY, 2002) <www-library.desy.de/diss02.html> (DESY-Thesis-2002-028).

18. Toellner, T. S. et al. Six-bounce $1 \mathrm{meV}$ monochromator for $23.7 \mathrm{keV}$ X-rays. (in the press).

19. Caticha, A. \& Caticha-Ellis, S. Dynamical theory of X-ray diffraction at angles near $\pi / 2$. Phys. Rev. B 25, 971-983 (1982).

\section{Acknowledgements}

The studies were carried out in the framework of the LDRD on XFELOs at Argonne National Laboratory. We are grateful to K.-J. Kim for the stimulated interest and discussions. We are indebted to R. Winarski and T. Graber for the loan of the diamond crystals. T. Toellner and D. Shu are acknowledged for the development of the high-resolution X-ray monochromator crucial for the presented studies. We acknowledge support of N. Zhang and M. Dudley in white-beam topography studies. Work was supported by the US Department of Energy, Office of Science, Office of Basic Energy Sciences, under Contract No. DE-AC02-06CH11357. NSLS was supported under Contract No. DE-AC-02-98CH10886.

\section{Author contributions}

Y.V.S. planned and organized the experiments, analysed the data and wrote the paper. S.S. set up and carried out the main experiment, the white-beam topography studies and contributed to writing of the paper. X.H. carried out the white-beam topography studies. All authors have contributed to the experimental effort of this work.

\section{Additional information}

The authors declare no competing financial interests. Reprints and permissions information is available online at http://npg.nature.com/reprintsandpermissions. Correspondence and requests for materials should be addressed to Y.V.S. 